\title{
Plataformas comunicacionales del ciberbullying. Una aplicación empírica en dos colegios de la quinta región, Chile
}

\author{
Communication Platforms of Cyberbullying. An Empirical Application \\ in Two Schools in the Fifth Region, Chile \\ Plataformas de comunicação de cyberbullying. Uma aplicação empírica \\ em duas escolas na quinta região do Chile
}

\section{Lorena Menay-López, ${ }^{a}$ Hanns de la Fuente-Mella. ${ }^{b}$}

a The Mackay School, Departamento de Ciencias Sociales y Filosofía. Avenida Vicuña Mackenna 700, Reñaca, Viña del Mar, Chile. Fono: 56-32-2386600. Correo electrónico: 1menay@ mackay.cl

${ }^{\text {b }}$ Pontificia Universidad Católica de Valparaíso, Facultad de Ciencias Económicas y Administrativas, Escuela de Comercio. Avenida Brasil 2830, 6 Piso, Valparaíso, Chile. Fono: 56-32-2273333.

Correo electrónico: hanns.delafuente@ucv.cl

\begin{abstract}
RESUMEN
El ciberbullying o acoso cibernético se ha transformado en uno de los fenómenos sociales más comunes en la comunidad escolar, donde el uso de plataformas electrónicas para cometer las agresiones hace que este tipo de acoso posea características propias. El objetivo de la presente investigación es caracterizar las plataformas comunicacionales que son utilizadas en el ciberbullying por los estudiantes de enseñanza básica de dos colegios de diferente estrato socioeconómico de la quinta región, Chile. Los resultados de la investigación muestran que tanto para el establecimiento socioeconómico alto como para el establecimiento socioeconómico medio, la plataforma comunicacional más utilizada por los estudiantes de enseñanza básica para hacer ciberbullying es Facebook; mientras que las víctimas señalan que el medio por el cual reciben este tipo de acoso es también Facebook para el caso de los estudiantes del establecimiento socioeconómico medio, y la telefonía celular para los estudiantes del establecimiento socioeconómico alto.
\end{abstract}

Palabras clave: ciberbullying, convivencia escolar, plataformas comunicacionales.

\begin{abstract}
The cyberbullying has become one of the most common social phenomena in the school community; the use of electronic platforms to commit aggression makes this type of harassment possess some particular characteristics. The aim of this research is to characterize the communication platforms used in cyberbullying by students of elementary education schools from two different socioeconomic strata of the fifth region, Chile. The research results show that for both, the high socioeconomic school and the medium socio-economic school, the most used communication platform for cyberbullying (by elementary school students) is Facebook. Victims indicate that the means by which they receive this type of harassment is Facebook for students of medium socio-economic school, and also cellular phones for students of a high socioeconomic setting.
\end{abstract}

Key words: cyberbullying, school life, communication platforms.

\section{RESUMO}

O cyberbullying ou cyberstalking tornou-se um dos fenômenos sociais mais comuns na comunidade escolar, onde a utilização de plataformas eletrônicas para cometer a agressão faz com que este tipo de assédio possua características. Objetivou-se caracterizar as plataformas de comunicação que são usadas em cyberbullying por alunos de escolas de Educação Básica em dois diferentes estratos socioeconômicos da região quinto, Chile. 
Estudios Pedagógicos XL, $\mathrm{N}^{\circ}$ 2: 117-133, 2014

PLATAFORMAS COMUNICACIONALES DEL CIBERBULLYING. UNA APLICACIÓN EMPÍRICA EN DOS COLEGIOS

DE LA QUINTA REGIÓN, CHILE

Resultados mostraram que tanto para a configuração de alto nível socioeconômico como o médio, a plataforma de comunicação mais utilizada para o cyberbullying pelos alunos do Ensino Fundamental é o Facebook; as vítimas, estudantes de configuração socioeconômico médio e telefones celulares para estudantes de ajuste socioeconômico alto, indicaram que o meio pelo qual elas recebem este tipo de assédio é também o Facebook.

Palavras chave: cyberbullying, vida escolar, plataformas de comunicação.

\section{INTRODUCCIÓN}

El ciberbullying, conocido también como acoso cibernético, se ha transformando en uno de los fenómenos más comunes en las comunidades escolares actuales. Algunos autores lo definen como la posibilidad de uso de las nuevas tecnologías de la información y de la comunicación para hostigar con ensañamiento a una víctima (Hernández y Solano, 2007), o como una manifestación del acoso escolar que se produce mediante plataformas virtuales y herramientas tales como: chats, blogs, fotologs, mensajes de texto para aparatos celulares, correo electrónico, foros, servidores que almacenan videos o fotografías, páginas web, teléfonos celulares y otros medios tecnológicos (Collell y Escudé, 2004).

Este tipo de bullying incluye todas aquellas conductas de burla, difamación, agresión, amedrentamiento e intimidación en contra de un individuo o grupo a través de teléfonos móviles o Internet (blogs, chat, correos, Facebook, etc.), lo cual tiene enormes consecuencias psicológicas en los afectados que ven totalmente vulnerada su identidad en su entorno social.

Aunque el ciberbullying puede ser considerado como una nueva modalidad o subtipo del bullying (Campbell, 2005), el uso de medios electrónicos para acometer las agresiones hace que este tipo de acoso tenga características distintas y propias, principalmente relacionadas con el anonimato del agresor. La mayoría de las veces, el agresor utiliza pseudónimos o nombres falsos para acosar a la víctima. La ocultación de la identidad facilita, por una parte, la agresión e impunidad del agresor y, por otra, aumenta el potencial de indefensión de la víctima (Monks et al., 2009).

El ciberbullying como problema lo podemos resumir en la sensación de impunidad que genera este fenómeno especialmente en las víctimas, ya que al operar con medios tecnológicos utilizables en cualquier momento, lugar y por cualquier tipo de adolescente, hace muy dificultoso su seguimiento. Por lo mismo, las comunidades escolares desconocen en gran medida qué disposiciones preventivas tomar ante una situación que no necesariamente surge dentro del colegio (Smith, 2006), lo cual ha generado intensos debates de quienes son los que deben responsabilizarse de este fenómeno social.

La presente investigación busca identificar las plataformas comunicacionales más habituales utilizadas en el ciberbullying por los alumnos de enseñanza básica (quintooctavo básico) en dos colegios de diferente estrato socioeconómico de la quinta región. Así, los objetivos que se persiguen son:

- Objetivo 1: Caracterizar las plataformas comunicacionales usadas por los alumnos de enseñanza básica del colegio de nivel socioeconómico medio en el ciberbullying.

- Objetivo 2: Caracterizar las plataformas comunicacionales usadas por los alumnos de enseñanza básica del colegio de nivel socioeconómico alto en el ciberbullying.

- Objetivo 3: Determinar si la variable nivel socioeconómico presenta diferencia en el ciberbullying escolar de enseñanza básica de estos dos colegios.

- Objetivo 4: Determinar si el grado de acceso a las tecnologías de la información incide en la práctica del ciberbullying escolar de enseñanza básica del colegio de nivel socioeconómico medio. 
- Objetivo 5: Determinar si el grado de acceso a las tecnologías de la información incide en la práctica del ciberbullying escolar de enseñanza básica del colegio de nivel socioeconómico alto.

Para el levantamiento de la información, la investigación utiliza una metodología mixta (cualitativa, cuantitativa), que involucra entrevistas previas a los equipos de orientación de cada colegio, los cuales entregan información necesaria para realizar una validación posterior del análisis cuantitativo.

Así, a través de la presente investigación será posible caracterizar las plataformas comunicacionales usadas en el ciberbullying por los alumnos de los colegios en estudio y, además, determinar si las variables nivel socioeconómico y grado de acceso a las tecnologías de la información, inciden en la práctica del ciberbullying escolar.

\section{MARCO TEÓRICO}

El hostigamiento, matonaje o acoso escolar también conocido por su término inglés bullying, se define como cualquier forma de maltrato psicológico, verbal o físico producido entre escolares de forma reiterada a lo largo de un tiempo determinado. Este fenómeno es de carácter transversal considerando que se produce en todas las clases sociales, en colegios públicos y privados, afectando a niños y niñas (Collell y Escudé, 2004).

En estos últimos años con la irrupción masiva de los medios tecnológicos (Castells, 1999), se nos presenta un nuevo tipo de abuso denominado ciberbullying, el cual utiliza las plataformas comunicacionales para realizar un tipo de agresión más expansiva y virulenta, lo que convierte a este problema social en un fenómeno mucho más preocupante que el bullying propiamente tal, por el nivel de alcance que éste tiene.

En el ciberbullying la víctima no tiene por qué ser un compañero de clase o un vecino del barrio, puede ser cualquiera al que se llegue a través de plataformas comunicacionales. Quien abusa no tiene porqué ser grande, ni fuerte, ni valiente, ni contar con el asentimiento del grupo, ni estar protegido por terceros. En este contexto que exige tan pocas condiciones a las partes intervinientes las posibles combinaciones son enormes.

A continuación se presenta una comparación entre las principales características del Bullying y el Ciberbullying (ver Tabla 1):

Tabla 1. Diferencias entre Bullying y Ciberbullying

\begin{tabular}{|c|c|}
\hline Bullying & Ciberbullying \\
\hline Cara a cara & Anónimo \\
\hline Es un grupo o individual & Individualmente \\
\hline Golpes, empujones, agresión verbal o exclusión social & Mensajes, $e$-mail, imágenes manipuladas \\
\hline Sólo en horas de escuela & En todos lados y a toda hora \\
\hline Se limita a la agresión directa & Sin límite de alcance \\
\hline Sólo audiencia escolar & Audiencia mundial por Internet \\
\hline Certeza de que sólo se encuentra en el ámbito escolar & $\begin{array}{c}\text { Incertidumbre de no saber quién ha visto } \\
\text { las imágenes o mensajes }\end{array}$ \\
\hline Víctima y agresor; víctima-agresor & Cibervíctima- Ciberagresor \\
\hline
\end{tabular}

Fuente: Joffre et al. (2011). 


\subsection{CARACTERÍSTICAS DEL CIBERBULLYING}

Respecto al tipo de agresiones electrónicas, Willard (2004) propone una clasificación de las mismas según la conducta realizada por el agresor. Las principales categorías son:

- Hostigamiento (envío y difusión de mensajes ofensivos o vulgares).

- Persecución (envío de mensajes amenazantes).

- Denigración (difusión de rumores sobre la víctima).

- Violación de la intimidad (difusión de secretos o imágenes de la víctima).

- Exclusión social (exclusión deliberada de la víctima de grupos en la red).

- Suplantación de la identidad (envío de mensajes maliciosos haciéndose pasar por la víctima).

En el ciberbullying se utilizan distintas plataformas comunicacionales con el objetivo de hostigar con ensañamiento a una víctima. Las herramientas disponibles en Internet ayudan a la propagación de ese comportamiento en el que las víctimas reciben malos tratos de sus iguales, sea a través de ridiculizaciones, amenazas, chantajes, discriminaciones, todo ello de manera anónima.

A continuación profundizaremos en algunas plataformas de ciberbullying descritas por Belsey (2005):

a) Teléfono Móvil (celular): Esta modalidad de ciberbullying adopta diversas formas según las posibilidades que permita el teléfono celular. La forma más tradicional son las llamadas y puede tener cabida no sólo desde la telefonía móvil sino desde cualquier teléfono fijo. El teléfono celular se ha convertido en un medio propicio para acosar, y la población joven o infantil que lo utiliza son los usuarios idóneos para ser acosados; averiguar un número de teléfono móvil es sencillo, así como ocultar el número del emisor de la llamada, con lo cual el anonimato queda plenamente garantizado. En ocasiones utilizan los móviles o números de otras personas, para evitar ser descubiertos, lo que demuestra no sólo la intencionalidad de los actos, sino también la improcedencia o inadecuación ética de los mismos. Asimismo, se trata de un medio que permite no sólo enviar mensajes orales, sino también textuales y multimedia, por tanto, la intimidación y la amenaza están garantizadas por cualquiera de las vías que se utilice.

b) Correo Electrónico: Es una de las herramientas de comunicación cuyo uso se ha extendido masivamente, considerando que en la actualidad que la mayoría de los jóvenes y niños disponen de una cuenta de correo electrónico, incluso antes de tener computador en casa. Esta forma de ciberbullying consiste en enviar repetidamente mensajes de correos electrónicos ofensivos y hostigadores a la persona que han convertido en víctima de sus abusos. Generalmente, se puede averiguar la cuenta de $e$-mail desde donde se enviaron los mensajes (lugar físico), siendo más fácilmente localizadas las cuentas locales o regionales que las grandes cuentas de e-mail como Hotmail, Gmail o Yahoo, pero en cualquier caso, difícilmente se puede conocer quién utilizó realmente esa cuenta.

c) Chats (mensajería instantánea): El uso que los niños y adolescentes hacen de la mensajería instantánea ha sido vinculado con el uso del teléfono, del $e$-mail, de las conversaciones cara a cara, centrándose excesivamente en estas cuestiones y descuidando otras problemáticas de mayor envergadura y relevancia (Grinter \& Palen, 2002). La realidad devela que esta tecnología ha tenido una gran acogida entre los 
jóvenes, quienes a pesar de utilizar otras formas de comunicación asincrónica, abogan por una interacción inmediata con el otro, en el que sus preguntas obtienen respuesta al instante. Así, el estudio de Grinter \& Palen (2002) concluye que el modo en que la gente joven utiliza la mensajería instantánea se correlaciona significativamente con el nivel de autonomía de los alumnos, convirtiéndose así en una herramienta propicia para la realización de ciberbullying.

d) Grabación de Violencia: Existe un tipo de agresor que disfruta grabando los golpes o intimidaciones que realiza a sus víctimas a través de sus teléfonos celulares, cámaras de video digital y después lo divulgan por la red. Ya no sólo disfruta acosando, sino que además quiere inmortalizar el acto en imagen digital, y obtener reconocimiento y respeto con ello. Los videos pueden colgarse en una web para ser vistos por un número indeterminado de personas, los cuales viralizan su contenido en forma masiva.

e) Desprestigio en la Web: Una de las mayores herramientas tecnológicas para desprestigiar, acosar o intimidar a las personas son las páginas web conocidas comúnmente como redes sociales, tales como: Facebook, Twitter, My Space, Blogspot, Fotolog y Flickr, entre otras. Éstas comparten una característica en común que suele ser bien aprovechada por los acosadores: la facilidad de acceso a cualquier persona de integrarse en estas redes suplantando datos y creándose un usuario falso, lo que sirve de mucho al ciberacosador para recopilar datos y observar la conducta de la víctima, además de extraer fotos sin su consentimiento de su red social. Otra característica de estas redes sociales es que el ciberacosador puede analizar las conductas que la persona posee con sus amistades con solamente observar el muro (Facebook) o libro (Fotolog), donde hayan conversaciones entre la víctima y uno de sus amigos, con la finalidad de poseer un perfil psicológico de ella, para en algún momento suplantar su identidad y desprestigiarlo frente a sus amigos (Hernández y Solano, 2007).

La naturaleza del acoso y las acciones que llevan a cabo los agresores a través de las nuevas tecnologías de la comunicación han hecho que las víctimas del acoso cibernético sufran los mismos efectos negativos sobre su salud mental y física que los agredidos por el bullying: baja autoestima, pobres resultados académicos, depresión, desajustes emocionales, desórdenes alimenticios, enfermedades crónicas, abandonos de domicilios y, en algunos casos, comportamientos violentos y suicidio (Slonje \& Smith, 2008).

Muchas víctimas sufren depresión (Hawker \& Boulton, 2000) la que permanece en la edad adulta (Olweus, 1998, 1993). Así, es crucial parar el acoso escolar para reducir y prevenir sus consecuencias negativas a corto y largo plazo. Ahora bien, respecto al perfil de las víctimas, Leonardo Cervera (2009) subraya que cualquiera está expuesto a este fenómeno aunque explica que los ciberacosadores suelen centrarse en aquellos niños que son diferentes "física o anímicamente".

\subsection{CONSUMO INFANTIL DE PLATAFORMAS COMUNICACIONALES}

Como consecuencia del crecimiento que han experimentado durante las últimas décadas los medios de comunicación electrónicos, tales como computadores, teléfonos móviles (celulares) y todo tipo de pantallas interactivas, se han realizado variados estudios en distintos países acerca de los hábitos de consumo de medios y sus posibles efectos. Muchos de estos estudios concluyen que los menores de edad, y entre ellos los pre-adolescentes, 
integran el grupo poblacional más dispuesto para adoptar y adaptar con mayor naturalidad estas nuevas herramientas comunicativas en su vida diaria (Bercedo et al., 2005; Del Río et al., 2010), encontrando las tecnologías de la información y la comunicación en este grupo un terreno especialmente abonado para su rápida implantación (Bringué, 2008; Flores, 2008).

Los efectos que las plataformas comunicacionales tienen sobre la audiencia son variados. De hecho, tienen influencias tanto positivas como negativas en los niños y adolescentes (Bringas et al., 2008), dependiendo de las características personales y contextuales de éstos, así como del propio contenido mediático o del tiempo de exposición a estas plataformas.

Dentro de las influencias positivas del consumo de medios electrónicos y utilización de plataformas comunicacionales se encuentra el fácil acceso a todo tipo de información, en especial el uso de Internet, el cual se caracteriza por ser un canal de comunicación inmediata, e imprescindible para difundir información y contactar con cualquier persona o institución en el mundo.

Por otra parte, existen influencias negativas asociadas al consumo de medios electrónicos y utilización de plataformas comunicacionales, lo que se traduce en que si bien estas herramientas fueron creadas como tecnologías para informar y comunicar, su propio diseño es susceptible de afectar la voluntad de control, lo que junto con otros factores personales y ambientales, facilita conductas adictivas, en especial en la adolescencia (Funk y Buchman, 1995; Funk, 1993).

\subsection{CIBERBULLYING EN EL MARCO DE LA CONVIVENCIA ESCOLAR}

La convivencia escolar constituye uno de los desafíos más importantes de la actualidad educativa; los colegios deben servir como espacios de aprendizaje donde se interioricen los hábitos y actitudes positivas de todos los integrantes de la comunidad educativa, en especial de los estudiantes. Así, es necesario entender la convivencia escolar no únicamente como un fin, pues también la debemos considerar como un medio, en el sentido en que puede convertirse en un recurso educativo que posibilite la adquisición de los valores propios de toda sociedad democrática (Campo, 2000).

Es importante destacar que en nuestro país, a partir de septiembre del 2011, se promulgó la nueva Ley de Violencia Escolar, que tiene la finalidad de regular y sancionar a aquellos que incurran en agresiones hacia sus pares, de manera de establecer mejores relaciones interpersonales y una sana convivencia escolar (Sistema de Información Legislativa, 2007).

Esta Ley de Violencia Escolar se ha convertido en un importante apoyo para los establecimientos educacionales en relación al problema del ciberbullying, considerando que esta ley regula normativamente las relaciones entre pares, y supone por primera vez dentro de la convivencia escolar la variable de los medios tecnológicos para cometer este tipo de agresiones.

\section{METODOLOGÍA}

Respecto a la metodología utilizada para alcanzar los objetivos propuestos, en la presente investigación se realiza un análisis cualitativo y cuantitativo fundamentado en fuentes de información primaria. El primero de ellos se basa en entrevistas ${ }^{1}$ realizadas al jefe de ciclo y al psicólogo del área del establecimiento socioeconómico alto, y a la

Las entrevistas no han sido grabadas por expresa solicitud de los entrevistados. 
directora del establecimiento socioeconómico medio, y se utiliza sólo como una manera de validar los resultados obtenidos del segundo análisis metodológico. El análisis cuantitativo se basa en una encuesta de 15 preguntas que se aplica a los dos colegios donde se realiza el análisis empírico de esta investigación ${ }^{2}$.

Los colegios elegidos corresponden a un establecimiento municipal subvencionado de Valparaíso y un colegio particular de Viña del Mar. Estos establecimientos poseen diferencias en su nivel socioeconómico, que se evidencian en el cobro de sus respectivas mensualidades. El colegio particular de Viña del Mar (denominado en adelante para esta investigación como el colegio de nivel socioeconómico alto) tiene actualmente una mensualidad que ronda los $\$ 220.000^{3}$, a lo cual se debe agregar una cuota de incorporación de 85 UF por estudiante 4 . Por otra parte, el colegio municipal subvencionado de Valparaíso (denominado en adelante para esta investigación como el colegio de nivel socioeconómico medio) tiene actualmente una mensualidad que va desde los \$10.000 a los \$ $15.000^{5}$, y no posee cuota de incorporación.

El análisis metodológico cuantitativo incluirá un análisis exploratorio descriptivo de frecuencias de cada una de las variables descritas en los objetivos, así como un análisis de la varianza de los grupos (ANOVA). A través del primero será posible diagnosticar las principales plataformas comunicacionales utilizadas por los alumnos de ambos colegios para realizar ciberbullying, e identificar si el grado de acceso a dichas plataformas posee influencia sobre la práctica del ciberbullying escolar y, a través del segundo, será posible realizar análisis comparativos intergrupos respecto a la variable nivel socioeconómico y su injerencia sobre el ciberbullying escolar.

Finalmente, y como una manera de validar los resultados obtenidos del análisis cuantitativo, éstos se contrastarán con los resultados obtenidos de las entrevistas realizadas a los grupos de estudio de cada uno de los colegios respectivos, lo que permitirá detectar las principales diferencias existentes entre las percepciones de los encargados de controlar este flagelo en los colegios y los actores del problema.

\subsection{ANÁLISIS CUALITATIVO}

El análisis cualitativo fundamentado en entrevistas se realiza a través de la metodología "entrevista personal", donde para el colegio del nivel socioeconómico medio el entrevistado fue la directora del establecimiento y el entrevistador fueron los autores de la investigación, mientras que para el colegio de nivel socioeconómico alto, el grupo de entrevista estaba conformado por dos personas (jefa de ciclo y psicólogo del área) y los autores de la investigación.

La entrevista fue diseñada en función de los objetivos de la investigación, sin embargo, y dado el alcance del análisis cualitativo, se abordaron dos preguntas asociadas a la investigación:

1. ¿Cree usted que los estudiantes de enseñanza básica del colegio en el que se desempeña realizan o son víctimas de ciberbullying?

2. ¿Considera usted que la variable nivel socioeconómico tendría algún nivel de injerencia en el ciberbullying escolar?

\footnotetext{
Para detalles de la encuesta contactar a los autores.

Considerando 10 cuotas al año.

Unidad de Fomento, Septiembre 2012, \$22.574,51.

Considerando 10 cuotas al año.
} 


\subsubsection{Resultados análisis cualitativo colegio nivel socioeconómico medio}

Respecto a la primera pregunta del análisis cualitativo es posible afirmar, en base a las respuestas obtenidas de la entrevista, lo siguiente: la encargada directa de manejar y controlar este flagelo afirma que entre los estudiantes de enseñanza básica de su colegio sí existe la figura del ciberbullying, afirmando tanto que existen grupos que lo comenten como víctimas del mismo. Además, la directora del establecimiento menciona que de acuerdo a su percepción de las denuncias realizadas, tanto las víctimas como los victimarios de ciberbullying utilizarían como plataforma principal Facebook, seguido por la telefonía móvil, que el grupo correspondería principalmente a estudiantes de los primeros años de enseñanza básica $\left(5^{\circ}\right.$ y $6^{\circ}$ año), y que principalmente serían mujeres. La directora señala que se implementarán medidas con el objeto de controlar el ciberbullying, sin embargo, por el momento las denuncias no son muchas, y que como política interna del establecimiento, cuando se recibe una denuncia que es sostenida en el tiempo, ella recomienda a los actores involucrados solucionar directamente el problema con la Policía de Investigaciones de Chile (PDI), esto último, basándose en que:

[...] el ciberbullying es muy difícil que lo cometan desde los propios computadores del establecimiento ${ }^{6}$, lo anterior fundamentado en que el establecimiento posee un estricto control sobre el registro de lo realizado por cada estudiante que utiliza los computadores del colegio, por lo que el uso de las plataformas comunicacionales para realizar ciberbullying es externo al establecimiento educacional.

Ahora bien, respecto al número de víctimas y/o victimarios, la directora del establecimiento no se atreve a entregar una cifra respecto al número de involucrados como víctimas, ni tampoco el número de los grupos victimarios, así como tampoco cuál de ambos sería mayor, dado que, entre otras razones, las denuncias por parte de estudiantes como de apoderados no son tan importantes en cuantía como en frecuencia, aunque la entrevistada admite que el ciberbullying en su establecimiento va en constante aumento.

En relación a la segunda pregunta de investigación formulada, la directora del establecimiento señala que de acuerdo a su percepción, no debieran existir diferencias entre el grado de ciberbullying realizado por estudiantes de enseñanza básica de colegios de diferentes niveles socioeconómicos, dado el acceso que hoy en día poseen los estudiantes a las plataformas comunicacionales, sin embargo, cree que las plataformas comunicacionales utilizadas podrían variar entre los diferentes establecimientos mencionados en la pregunta, sosteniendo la idea de que en el colegio que ella dirige la principal plataforma comunicacional utilizada para realizar ciberbullying sería Facebook.

\subsubsection{Resultados análisis cualitativo colegio nivel socioeconómico alto}

En relación a la primera pregunta del análisis cualitativo realizado es posible afirmar, en base a las respuestas obtenidas de la entrevista, lo siguiente: los encargados de manejar

Hace referencia a computadores como principal plataforma dado que la percepción de la entrevistada es que el ciberbullying se realiza a través de Facebook, y los estudiantes de este establecimiento no tendrían un elevado acceso a los smartphone desde donde tendrían también acceso a Facebook. 
y controlar este flagelo afirman que entre los estudiantes de enseñanza básica de su colegio sí existe ciberbullying, afirmando tanto que existen grupos que lo comenten como víctimas del mismo, sin embargo, existen ciertas diferencias al momento de señalar si efectivamente es un problema grave en términos del número de afectados, o bien sólo es un tema que social y mediáticamente se ha exacerbado. Por lo mismo, no existe un consenso en cuanto a una cifra cierta respecto al número de involucrados como víctimas ni tampoco el número de los grupos victimarios, no obstante, admiten que las denuncias por parte de los apoderados de estudiantes víctimas de ciberbullying ha ido incrementando en el tiempo, no así el número de denuncias por parte de los estudiantes involucrados. El grupo entrevistado señala que constantemente se realizan medidas paliativas con el objeto de controlar el ciberbullying, principalmente focalizadas en charlas informativas dictadas por el orientador del colegio.

En relación a la segunda pregunta de investigación formulada, los participantes de esta entrevista señalan que, de acuerdo a su percepción, debieran existir diferencias entre el grado de ciberbullying realizado por estudiantes de enseñanza básica de colegios de diferentes niveles socioeconómicos, dado que a pesar de que el acceso que hoy en día poseen los estudiantes a las plataformas comunicacionales es amplio, hacen expresa la diferencia en términos del costo que tiene cada una de estas plataformas, lo que limitaría el acceso a las mismas en los grupos socioeconómicos educacionales medios o bajos.

\subsection{ANÁLISIS CUANTITATIVO}

La primera etapa del análisis cuantitativo consiste en la aplicación de una encuesta basada en escalas cualitativas de categorías dicotómicas y en escalas múltiples, dirigida tanto a los estudiantes de enseñanza básica del establecimiento socioeconómico medio como alto. Para el cálculo del tamaño muestral se utiliza la fórmula propuesta por Martínez (1994):

$$
n=\frac{Z^{2} p q N}{N E^{2}+Z^{2} p q}
$$

Donde,

- $\quad$ N: tamaño de la población (238 Colegio Socioeconómico Medio; 286 Colegio

Socioeconómico Alto, correspondiente a todos los alumnos de enseñanza básica).

- E: error absoluto admisible (10\%).

- p, q: cada una tiende al $50 \%$.

- n: tamaño muestral (68 Colegio Socioeconómico Medio; 72 Colegio Socioeconómico Alto).

No obstante, y con el objeto de homogeneizar las muestras, se ha trabajado para ambos establecimientos con el mismo tamaño muestral, es decir, 72 encuestas para el establecimiento socioeconómico medio y 72 encuestas para el establecimiento socioeconómico alto, mejorando así el error absoluto admisible para el establecimiento socioeconómico medio en 0,4\%, además, se ha considerado para el análisis la metodología de muestreo estratificado simple.

Una vez diseñada la encuesta se ha realizado un pre-test al $10 \%$ de la muestra, con el objeto de validar la fiabilidad del instrumento. Del pre-test realizado, así como de las entrevistas realizadas en ambos establecimientos educacionales, ha surgido la necesidad de replantear el orden inicial del instrumento, dado que las preguntas asociadas al hecho de ser victimario o víctima de ciberbullying se encontraban al inicio de la encuesta, por lo 
Estudios Pedagógicos XL, N 2: 117-133, 2014

PLATAFORMAS COMUNICACIONALES DEL CIBERBULLYING. UNA APLICACIÓN EMPÍRICA EN DOS COLEGIOS

DE LA QUINTA REGIÓN, CHILE

que el resto de las preguntas asociadas a las plataformas más utilizadas por los estudiantes quedaban sesgadas y supeditadas a estas iniciales preguntas, de esta manera, se decide poner las preguntas referidas al final del cuestionario.

\subsubsection{Resultados análisis cuantitativo colegio nivel socioeconómico medio}

El objeto de este análisis es dar respuesta a los siguientes objetivos planteados en la investigación:

- Objetivo 1: Caracterizar las plataformas comunicacionales usadas por los alumnos de enseñanza básica del colegio de nivel socioeconómico medio en el ciberbullying.

- Objetivo 4: Determinar si el grado de acceso a las tecnologías de la información incide en la práctica del ciberbullying escolar de enseñanza básica del colegio de nivel socioeconómico medio.

Para dar cumplimiento a estos objetivos se realizaron cinco preguntas: i) marca con un círculo si tienes alguno de los siguientes medios electrónicos para tu uso personal (televisión, radio, computador fijo, notebook, IPad, teléfono celular, smartphone), ii) ¿has recibido mensajes o fotografías ofensivas a tu teléfono celular?, iii) ¿has enviado mensajes o fotografías ofensivas desde tu teléfono celular?, iv) ¿has participado en grupos ofensivos hacia algún compañero de colegio desde tu cuenta de Facebook?, v) ¿has sido víctima de algún grupo en contra tuya en Facebook? Si bien la primera pregunta no plantea directamente la relación entre plataforma comunicacional y ciberbullying, ésta es utilizada como variable proxy de las herramientas que poseen los alumnos de los establecimientos que potencialmente podrían ser utilizadas con este objetivo.

La primera pregunta nos permite caracterizar si el grado de acceso a las tecnologías de la información incide en la práctica del ciberbullying de los estudiantes de enseñanza básica del establecimiento educacional socioeconómico medio. De la Tabla 2 es posible observar la elevada presencia de medios electrónicos como teléfono celular y la televisión en los estudiantes de este establecimiento. Respecto a la pertenencia de computadores, ya sea fijos o bien notebook en el establecimiento analizado, el porcentaje varía igualmente entre ellos siendo un 33\% para cada uno de estos medios electrónicos. Finalmente, la presencia de IPad o smartphone en los estudiantes de enseñanza básica del establecimiento socioeconómico medio es casi inobservable (6\% y 10\%, respectivamente). Por lo anterior, podría concluirse que en este tipo de establecimiento el acceso a las tecnologías asociadas a la telefonía celular y a los computadores (fijos o notebook) podrían considerarse como los principales determinantes del ciberbullying.

Tabla 2. Porcentajes de medios electrónicos de uso personal establecimiento socioeconómico medio

\begin{tabular}{|c|c|c|c|c|c|c|c|}
\hline $\begin{array}{c}\text { Establecimiento } \\
\text { Socioeconómico } \\
\text { Medio }\end{array}$ & Televisión & Radio & $\begin{array}{c}\text { Computador } \\
\text { Fijo }\end{array}$ & Notebook & IPad & $\begin{array}{c}\text { Teléfono } \\
\text { Celular }\end{array}$ & Smartphone \\
\cline { 2 - 8 } & $72 \%$ & $44 \%$ & $33 \%$ & $33 \%$ & $6 \%$ & $74 \%$ & $10 \%$ \\
\hline
\end{tabular}

Fuente: elaboración propia. 
En relación al uso de la telefonía celular tanto para recibir o bien enviar mensajes o fotografías ofensivas, un $23 \%$ de los estudiantes de enseñanza básica del establecimiento socioeconómico medio señala que sí ha recibido mensajes de esta naturaleza a través de este medio electrónico, y un $11 \%$ señala que ha utilizado la telefonía celular para enviar mensajes ofensivos. Respecto a la participación en grupos ofensivos utilizando la plataforma Facebook, un $17 \%$ de los estudiantes de enseñanza básica del establecimiento socioeconómico medio señala que ha participado en esta actividad, mientras que un $29 \%$ indica haber sido víctima de algún grupo en contra suya a través de Facebook (ver Tabla 3).

Tabla 3. Porcentajes víctimas-victimarios según plataforma comunicacional

\begin{tabular}{|c|c|c|c|}
\hline \multirow{2}{*}{$\begin{array}{c}\text { Establecimiento } \\
\text { Socioeconómico } \\
\text { Medio }\end{array}$} & Plataforma & Victimario & Víctima \\
\cline { 2 - 4 } & Teléfono Celular & $11 \%$ & $23 \%$ \\
\cline { 2 - 4 } & Facebook & $17 \%$ & $29 \%$ \\
\hline
\end{tabular}

Fuente: elaboración propia.

En conclusión, la plataforma comunicacional más utilizada por los estudiantes de enseñanza básica del colegio de nivel socioeconómico medio para hacer ciberbullying es Facebook por sobre el uso de la telefonía celular, mientras que las víctimas de ciberbullying señalan que el medio por el cual reciben este tipo de ataque es también preferentemente a través de esta plataforma. En ambos casos tanto para las víctimas como para los victimarios la diferencia en el uso de las plataformas comunicacionales corresponde a un $6 \%$ entre el uso de Facebook y telefonía celular. Además, siempre el porcentaje de las víctimas de ciberbullying a través de ambas plataformas supera a los victimarios (12\% más de víctimas).

Los anteriores resultados son posibles comprobarlos a través de la pregunta realizada en la encuesta: ¿cuánto tiempo aproximado dedicas diariamente a tu Facebook? Esta pregunta nos ayuda a validar lo referente a la caracterización de la plataforma comunicacional más utilizada por los alumnos de enseñanza básica para el ciberbullying en lo que a frecuencia de uso se refiere (ver Tabla 4).

Tabla 4. Porcentaje de tiempo diario dedicado al uso de Facebook

\begin{tabular}{|c|c|c|c|c|}
\hline \multirow{2}{*}{$\begin{array}{c}\text { Establecimiento } \\
\text { Socioeconómico } \\
\text { Medio }\end{array}$} & $\begin{array}{c}\text { Menos de una } \\
\text { hora }\end{array}$ & $\begin{array}{c}\text { Aproximadamente } \\
\text { una hora }\end{array}$ & $\begin{array}{c}\text { Dos a tres } \\
\text { horas }\end{array}$ & $\begin{array}{c}\text { Mucho más de 3 } \\
\text { horas }\end{array}$ \\
\cline { 2 - 4 } & $11 \%$ & $42 \%$ & $26 \%$ & $12 \%$ \\
\hline
\end{tabular}

Fuente: elaboración propia.

Así, de los resultados presentados en la Tabla 4 es posible concluir que la mayoría de los estudiantes de enseñanza básica del establecimiento socioeconómico medio utiliza la plataforma Facebook entre una hora, y dos a tres horas diariamente, caracterizando así la frecuencia de uso de la citada plataforma. 


\subsubsection{Resultados análisis cuantitativo colegio nivel socioeconómico alto}

Este análisis es para dar respuesta a los siguientes objetivos planteados en la investigación:

- Objetivo 2: Caracterizar las plataformas comunicacionales usadas por los alumnos de enseñanza básica del colegio de nivel socioeconómico alto en el ciberbullying.

- Objetivo 5: Determinar si el grado de acceso a las tecnologías de la información incide en la práctica del ciberbullying escolar de enseñanza básica del colegio de nivel socioeconómico alto.

Para entregar respuesta estos objetivos se realizaron cinco preguntas: i) marca con un círculo si tienes alguno de los siguientes medios electrónicos para tu uso personal (televisión, radio, computador fijo, notebook, IPad, teléfono celular, smartphone), ii) ¿has recibido mensajes o fotografías ofensivas a tu teléfono celular?, iii) ¿has enviado mensajes o fotografías ofensivas desde tu teléfono celular?, iv) ¿has participado en grupos ofensivos hacia algún compañero de colegio desde tu cuenta de Facebook?, v) ¿has sido víctima de algún grupo en contra tuya en Facebook? Si bien la primera pregunta no plantea directamente la relación entre plataforma comunicacional y ciberbullying, ésta es utilizada como variable proxy de las herramientas que poseen los alumnos de los establecimientos que potencialmente podrían ser utilizadas con este objetivo.

A través de la primera pregunta podemos caracterizar si el grado de acceso a las tecnologías de la información incide en la práctica del ciberbullying de los estudiantes de enseñanza básica del establecimiento educacional socioeconómico alto. De la Tabla 5 es posible observar la elevada presencia de medios electrónicos como la televisión, los IPad, los notebook y los smartphone en los estudiantes de este establecimiento. Respecto a la pertenencia de computadores, ya sea fijos o bien notebook en el establecimiento analizado, el porcentaje varía de forma diferente entre ellos siendo un 56\% para el caso de los notebook y de un 44\% para el caso de los computadores fijos. Por último, la posesión de un teléfono celular por parte de los estudiantes de enseñanza básica del establecimiento socioeconómico alto es reemplazado por la pertenencia de un smartphone (33\% y 40\%, respectivamente). Por lo anterior, podría concluirse que en este tipo de establecimiento el acceso a las tecnologías asociadas a los notebook, IPad y smartphone podría considerarse como el principal determinante del ciberbullying.

Tabla 5. Porcentajes de medios electrónicos de uso personal establecimiento socioeconómico alto

\begin{tabular}{|c|c|c|c|c|c|c|c|}
\hline $\begin{array}{c}\text { Establecimiento } \\
\text { Socioeconómico } \\
\text { Alto }\end{array}$ & Televisión & Radio & $\begin{array}{c}\text { Computador } \\
\text { Fijo }\end{array}$ & Notebook & IPad & $\begin{array}{c}\text { Teléfono } \\
\text { Celular }\end{array}$ & Smartphone \\
\cline { 2 - 7 } & $63 \%$ & $49 \%$ & $44 \%$ & $56 \%$ & $62 \%$ & $33 \%$ & $40 \%$ \\
\hline
\end{tabular}

Fuente: elaboración propia.

En lo que respecta al uso de la telefonía celular tanto para recibir o bien enviar mensajes o fotografías ofensivas, un $21 \%$ de los estudiantes de enseñanza básica del establecimiento socioeconómico alto señala que sí ha recibido mensajes de esta naturaleza a través de 
este medio electrónico, y un $10 \%$ señala que ha utilizado la telefonía celular para enviar mensajes ofensivos. En relación a la participación en grupos ofensivos utilizando la plataforma Facebook, un $17 \%$ de los estudiantes de enseñanza básica del establecimiento socioeconómico alto señala que ha participado en esta actividad, mientras que un $11 \%$ indica haber sido víctima de algún grupo en contra suya a través de Facebook (ver Tabla 6).

Tabla 6. Porcentajes víctimas-victimarios según plataforma comunicacional

\begin{tabular}{|c|c|c|c|}
\hline \multirow{2}{*}{$\begin{array}{c}\text { Establecimiento } \\
\text { Socioeconómico } \\
\text { Alto }\end{array}$} & Plataforma & Victimario & Víctima \\
\cline { 2 - 4 } & Teléfono Celular & $10 \%$ & $21 \%$ \\
\cline { 2 - 4 } & Facebook & $17 \%$ & $11 \%$ \\
\hline
\end{tabular}

Fuente: elaboración propia.

En conclusión, la plataforma comunicacional más utilizada por los estudiantes de enseñanza básica del colegio de nivel socioeconómico alto para hacer ciberbullying es Facebook por sobre el uso de la telefonía celular, mientras que las víctimas de ciberbullying señalan que el medio por el cual reciben este tipo de ataque es preferentemente a través de telefonía celular. La diferencia en el uso de las plataformas comunicacionales para las víctimas y victimarios corresponde a un $7 \%$ para los victimarios prefiriendo éstos el uso de Facebook y un $10 \%$ para las víctimas recibiendo el ataque preferentemente a través de los teléfonos celulares. Ahora bien, el porcentaje de las víctimas de ciberbullying a través de telefonía celular supera al porcentaje de los victimarios (11\% más de víctimas), sin embargo, el porcentaje de los victimarios de ciberbullying a través de Facebook supera al porcentaje de las víctimas (6\% más de victimarios).

Los anteriores resultados son posibles comprobarlos a través de la pregunta realizada en la encuesta: ¿cuánto tiempo aproximado dedicas diariamente a tu Facebook? Esta pregunta nos ayuda a validar lo referente a la caracterización de la plataforma comunicacional más utilizada por los alumnos de enseñanza básica para el ciberbullying en lo que a frecuencia de uso se refiere (ver Tabla 7).

Tabla 7. Porcentaje de tiempo diario dedicado al uso de Facebook

\begin{tabular}{|c|c|c|c|c|}
\hline \multirow{2}{*}{$\begin{array}{c}\text { Establecimiento } \\
\text { Socioeconómico }\end{array}$} & $\begin{array}{c}\text { Menos de una } \\
\text { hora }\end{array}$ & $\begin{array}{c}\text { Aproximadamente } \\
\text { una hora }\end{array}$ & $\begin{array}{c}\text { Dos a tres } \\
\text { horas }\end{array}$ & $\begin{array}{c}\text { Mucho más de 3 } \\
\text { horas }\end{array}$ \\
\cline { 2 - 5 } & $35 \%$ & $26 \%$ & $18 \%$ & $9 \%$ \\
\hline
\end{tabular}

Fuente: elaboración propia.

Así, de los resultados presentados en la Tabla 7 es posible concluir que la mayoría de los estudiantes de enseñanza básica del establecimiento socioeconómico alto utiliza la plataforma Facebook entre menos de una hora, y aproximadamente una hora diariamente, caracterizando así la frecuencia de uso de la citada plataforma. 


\subsection{ANÁLISIS COMPARATIVO ESTABLECIMIENTO SOCIOECONÓMICO MEDIO/ALTO}

El objeto de este análisis es dar respuesta al siguiente objetivo planteado en la investigación:

- Objetivo 3: Determinar si la variable nivel socioeconómico presenta diferencia en el ciberbullying escolar de enseñanza básica de estos dos colegios.

Para determinar la existencia de diferencias significativas entre los estudiantes de enseñanza básica de los dos colegios bajo estudio se realizaran test ANOVA para las preguntas asociadas al objeto de investigación planteado, éstas son: i) ¿has recibido mensajes o fotografías ofensivas a tu teléfono celular?, ii) ¿has enviado mensajes o fotografías ofensivas desde tu teléfono celular?, iii) ¿has participado en grupos ofensivos hacia algún compañero de colegio desde tu cuenta de Facebook?, iv) ¿has sido víctima de algún grupo en contra tuya en Facebook? Donde la hipótesis nula establece que existe igualdad en el ciberbullying escolar de enseñanza básica de los dos colegios de diferente nivel socioeconómico ${ }^{7}$.

Tabla 8. ANOVA igualdad de medias ciberbullying escolar establecimientos diferentes niveles socioeconómicos

\begin{tabular}{|l|c|c|c|}
\hline Ciberbullying / Plataforma & Valor t & P Value & Significatividad al 95\% \\
\hline Víctima / Teléfono Celular & 0,000 & 1 & Acepta \\
\hline Victimario / Teléfono Celular & 0,241 & 0,810 & Acepta \\
\hline Victimario / Facebook & $-0,217$ & 0,829 & Acepta \\
\hline Víctima / Facebook & 3,014 & 0,004 & Rechaza \\
\hline
\end{tabular}

Fuente: elaboración propia.

Respecto a lo anterior, y como se puede apreciar en la Tabla 8 del análisis ANOVA realizado, es posible observar que la única diferencia estadísticamente significativa respecto a la existencia de diferencias en el ciberbullying escolar en la enseñanza básica en los dos colegios analizados de diferentes niveles socioeconómicos es respecto a las víctimas asociadas a la plataforma Facebook, respecto a las otras formas de ciberbullying ya sea para las víctimas o para los victimarios, y respecto a las plataformas utilizadas para la realización de ciberbullying, no existen diferencias estadísticamente significativas entre ambos establecimientos educacionales.

\section{CONCLUSIONES, LIMITACIONES Y FUTUROS TRABAJOS}

Respecto a la pregunta de investigación del presente trabajo asociada a: ¿cuáles son las plataformas comunicacionales usadas en el ciberbullying por parte de los alumnos

\footnotetext{
Se han considerado las preguntas asociadas tanto a víctima como a victimario, dado que ambos forman parte del ciberbullying, además se hace la distinción de la plataforma comunicacional utilizada.
} 
de enseñanza básica de dos colegios de la quinta región?, y a los objetivos planteados, de los resultados obtenidos de la metodología es posible apreciar que en relación a: i) la caracterización de las plataformas comunicacionales usadas en el ciberbullying por los estudiantes de enseñanza básica de ambos colegios estudiados y, ii) si el grado de acceso a las tecnologías de la información incide en la práctica del ciberbullying escolar de enseñanza básica de los colegios analizados, en el establecimiento socioeconómico medio, las plataformas comunicacionales más utilizadas por los alumnos son la telefonía celular y los computadores ya sea de tipo fijo o notebook, respecto a las plataformas comunicacionales utilizadas por los alumnos en el ciberbullying en el establecimiento socioeconómico alto, las plataformas más utilizadas son los notebook, IPad y smartphone. Ahora bien, la plataforma comunicacional más utilizada por los estudiantes de enseñanza básica del colegio de nivel socioeconómico medio para hacer ciberbullying es Facebook por sobre el uso de la telefonía celular, mientras que las víctimas de ciberbullying señalan que el medio por el cual reciben este tipo de ataque es también preferentemente a través de Facebook. Por su parte, la plataforma comunicacional más utilizada por los estudiantes de enseñanza básica del colegio de nivel socioeconómico alto para hacer ciberbullying es Facebook por sobre el uso de la telefonía celular, mientras que las víctimas de ciberbullying señalan que el medio por el cual reciben este tipo de ataque es preferentemente a través de telefonía celular.

Lo anterior se valida a través de la frecuencia de uso diario de la plataforma Facebook, siendo ésta notoriamente superior en los estudiantes del establecimiento socioeconómico medio por sobre el establecimiento socioeconómico alto. Del análisis realizado, llama la atención el bajo porcentaje de estudiantes del establecimiento socioeconómico medio que no responde la encuesta $(1,22 \%)$, versus el alto porcentaje de estudiantes del establecimiento socioeconómico alto que no responde la encuesta $(12,20 \%)$.

En relación al análisis comparativo realizado en la presente investigación y que nos ha permitido determinar si la variable nivel socioeconómico presenta diferencias en el ciberbullying escolar de enseñanza básica de estos dos colegios, del análisis ANOVA realizado es posible concluir que no existen diferencias estadísticamente significativas entre las víctimas y victimarios del ciberbullying en lo que respecta al uso de la plataforma comunicacional telefonía celular, así como también para el caso de los victimarios en el uso de la plataforma Facebook, la única diferencia estadísticamente significativa se da para el caso de las víctimas acosadas a través de la plataforma comunicacional Facebook y telefonía celular, esto sucede dado que los estudiantes del establecimiento socioeconómico medio utilizan principalmente computadores como medio de conexión para Facebook, mientras que los estudiantes del establecimiento socioeconómico alto utilizan además de los computadores como medio de conexión para Facebook sus smartphones, que para esta investigación han sido considerados como una plataforma distinta a la telefonía celular.

En la presente investigación se ha mencionado que la metodología cualitativa sería utilizada como medio para validar los resultados obtenidos del análisis cuantitativo, así, es posible observar del análisis de las entrevistas realizadas tanto a la directora del establecimiento socioeconómico medio, como al grupo de entrevistados del establecimiento socioeconómico alto, que sus percepciones a pesar de ser diferentes respecto a lo que podría ocurrir en uno u otro establecimiento, no se encuentran muy alejadas de los resultados cuantitativos. Así, la directora del establecimiento socioeconómico medio señala que Facebook sería la principal plataforma comunicacional utilizada por los estudiantes de su colegio para el ciberbullying, lo que coincide con los resultados cuantitativos. Respecto 
a la pregunta asociada a si existen diferencias entre el grado de ciberbullying realizado por estudiantes de enseñanza básica de colegios de diferentes niveles socioeconómicos, la directora respalda las conclusiones obtenidas por el análisis cuantitativo, dado que según su percepción no debieran existir tales diferencias en el nivel de ciberbullying, estas diferencias sólo debieran producirse en las plataformas comunicacionales utilizadas. Ahora bien, de las entrevistas realizadas al grupo de profesionales del establecimiento socioeconómico alto, y respecto a la plataforma comunicacional más utilizada para el ciberbullying, los resultados obtenidos por la metodología cuantitativa son validados por la metodología cualitativa, dado que según los entrevistados no existe un consenso respecto a cuál podría ser la plataforma más usada, sin embargo, respecto a la pregunta asociada a si existen diferencias entre el grado de ciberbullying realizado por estudiantes de enseñanza básica de colegios de diferentes niveles socioeconómicos, el grupo entrevistado señala que es posible que existan tales diferencias, sin embargo, el análisis cuantitativo sólo acepta tales diferencias en lo que a uso de la plataforma comunicacional Facebook se refiere y sólo para el caso de las víctimas.

Esta investigación constituye los primeros pasos dentro de una línea más ambiciosa, por lo que se enfrenta a una serie de limitaciones e investigaciones futuras. Así, se plantea la posibilidad de repetir el estudio, considerando las mismas variables, para establecer la evolución del comportamiento de los estudiantes en el tiempo y de este modo poder generar un patrón que permita mejorar la toma de decisiones asociadas al ciberbullying escolar. Del mismo modo, y teniendo en cuenta que si bien todos los cursos están representados de forma proporcional en la muestra analizada, el tamaño de los mismos no es uniforme, sería interesante realizar un análisis para diferentes segmentos de la población de estudiantes, teniendo en cuenta variables como el género, el curso, etc., aunque ello llevase a ampliar el tamaño de cada una de las submuestras. También sería recomendable contemplar y analizar los puntos de vista de otros colegios, haciendo análisis comparativos entre colegios de otros niveles socioeconómicos, así como también considerar la opinión de los apoderados.

\section{REFERENCIAS BIBLIOGRÁFICAS}

Belsey, B. (2005). Cyberbullying: An Emerging Threats to the "Always On” Generation. Recuperado de http://www.cyberbullying.ca/pdf/Cyberbullying_Article_by_Bill_Belsey.pdf, consultado en julio de 2012.

Bercedo, A., Redondo, C., Pelayo, R., Gómez del Río, Z., Hernández, M. y Cadenas, N. (2005). Consumo de los medios de comunicación en la adolescencia. Anales de Pediatría, vol. 63, n. 6, 516-525.

Bringas, C., Ovejero, A., Herrero, F. J. y Rodríguez, F. J. (2008). Medios electrónicos y comportamiento antisocial en adolescentes. Revista Colombiana de Psicología, vol. 17, n. 1, 93-104.

Bringué, X. (2008). Niños y jóvenes en un nuevo escenario de comunicación. Actas del XXI Congreso Internacional de Comunicación (EUNSA), Pamplona, España (pp. 17-32). Recuperado de http://hdl.handle.net/10171/16876, consultado en julio de 2012.

Campo, A. (2000). De la convivencia escolar o de la reconstrucción de las expectativas. Organización y Gestión Educativa, n. 4, 3-8.

Campbell, M. (2005). Cyber Bullying: An Old Problem in a Bew Guise? Australian Journal of Guidance and Counselling, vol. 15, n. 1, 68-76.

Castells, M. (1999). Internet y la sociedad red. Lección inaugural del programa de doctorado sobre la sociedad de la información y el conocimiento (UOC). Recuperado de http://www.uoc.edu/ 
web/cat/articles/castells/castellsmain2.html, consultado en julio de 2012.

Cervera, L. (2009). Lo que hacen tus hijos en Internet: Una guía para padres. Barcelona: RBA Libros.

Collell, J. y Escudé, C. (2004). Rol de les emocions en els processos de maltractament entre alumnes. Àmbits de Psicopedagogia, n. 12, 21-26.

Del Río, J., Sádaba, C. y Bringué, X. (2010). Menores y redes ¿sociales?: De la amistad al ciberbullying. Revista de Estudios de Juventud, n. 88, 115-129.

Flores, J. (2008). Ciberbullying. Guía rápida. Recuperado de http://www.pantallasamigas.net/ proteccion-infancia-consejos-articulos/ciberbullying-guia-rapida.shtm, consultado en julio de 2012.

Funk, J. B. \& Buchman, D. (1995). Video Games Controversies. Pediatric Annals, vol. 24, n. 2, 91-94.

(1993). Reevaluating the Impact of Video Games. Clinical Pediatrics, vol. 32, n. 2, 86-90.

Grinter, R. E. \& Palen, L. (2002). Instant Messaging in Teenage Life. Proceedings of the 2002 ACM Conference on Computer Supported Cooperative Work (CSCW) (pp. 21-30). New Orleans, Louisiana, USA.

Hawker, D. \& Boulton, M. (2000). Twenty Years' Research on Peer Victimization and Psychosocial Maladjustment: A Meta-analytic Review of Cross-sectional Studies. Journal of Child Psychology and Psychiatry, vol. 41, n. 4, 441-455.

Hernández, M. A. y Solano, I. M. (2007). Ciberbullying, un problema de acoso escolar. Revista Iberoamericana de Educación a Distancia, vol. 10, n. 1, 17-36.

Joffre, V. M., García, G., Saldívar, A., Martínez, G., Lin, D., Quintanar, S. y Villasana, A. (2011). Bullying en alumnos de secundaria, características generales y factores asociados de riesgo. Boletín Médico del Hospital Infantil de México, vol. 68, n. 3, 193-202.

Martínez, C. (1994). Estadística comercial. Santa Fe de Bogotá: Grupo Editorial Norma Educativa.

Monks, C., Smith, P., Naylor, P., Barter, C., Ireland, J. \& Coyne, I. (2009). Bullying in Different Contexts: Commonalities, Differences, and the Role of Theory. Aggression and Violent Behavior, vol. 14, n. 2, 146-156.

Olweus, D. (1998). Conductas de acoso y amenaza entre escolares (2a Ed.). Madrid: Morata. . (1993). Bullying at School: What We Know and What We Can Do. Malden, MA: Blackwell Publishing.

Sistema de Información Legislativa. (2007). Boletín No 5521-07. Proyecto de ley que sanciona como delito de exhibición y distribución a través de Internet material que contenga conducta propia de maltrato escolar. Recuperado de http://sil.senado.cl/cgi-bin/index_eleg.pl?5521-07, consultado en julio de 2012.

Slonje, R. \& Smith, P. (2008). Cyberbullying: Another Main Type of Bullying? Scandinavian Journal of Psychology, vol. 49, n. 2, 147-154.

Smith, P. K. (2006). Ciberacoso: Naturaleza y extensión de un nuevo tipo de acoso dentro y fuera de la escuela. Congreso Educación Palma de Mallorca, España. Recuperado de http://www. observatorioperu.com/lecturas/ciberacoso_pSmith.pdf, consultado en julio de 2012.

Willard, N. (2004). An Educator's Guide to Cyberbullying and Cyberthreats. Center for Safe and Responsible Internet Use. Recuperado de http://www.wcs.k12.va.us/users/honaker/cyberbullyingfor-teachers.pdf, consultado en julio de 2012. 
